\title{
EFFECTS OF PROBIOTICS ON HUMAN HEALTH AND DISEASE: A REVIEW
}

\author{
A. Amiri', F. Firoozeh², M. Zibaei ${ }^{3}$, A. Khaledi ${ }^{4}$ \\ ${ }^{1}$ Faculty of Basic Sciences, Islamic Azad University, Sciences and Research Branch -Tehran, I.R. Iran \\ 2Dietary Supplements and Probiotic Research Center, Alborz University of Medical Sciences - Karaj, I.R. Iran \\ ${ }^{3}$ Evidence-based Phytotherapy and Complementary Medicine Research Center, \\ Alborz University of Medical Sciences - Karaj, Iran \\ ${ }^{4}$ Infectious Diseases Research Center, Kashan University of Medical Sciences - Kashan, I.R., Iran
}

\begin{abstract}
Alteration of the gut microbiome in order to achieve a balance in the normal flora of the intestine could be very beneficial in maintaining the health of the human. Probiotics are living microbial supplements that are added to the diet and have beneficial effects on the host by improving the balance of the intestinal microflora. The purpose of this study is to review previous studies on the effects of probiotics on human health and various diseases. The Farsi and English electronic databases such as, SID, Iranmedex, Magiran, Google Scholar, PubMed and ISI Web of Knowledge were searched and the published articles that have studied the effects of probiotics on the prevention and treatment of various diseases were included in the study. The review of published articles related to the subject showed that consumption of probiotics, prebiotics and proper diet have the significant effects on the health of the digestive system and has reduced and improved symptoms of different disorders and diseases. Further research is needed to better understand the underlying mechanisms of probiotic function and confirm the role of the probiotics in preventing and treating various types of cancers and other diseases.
\end{abstract}

Key words: probiotics, gut microbiota, human health, dysbiosis, intestinal diseases, extra-intestinal diseases

Corresponding author: Farzaneh Firoozeh Ph.D, Dietary Supplements and Probiotic Research Center, Alborz University of Medical Sciences, Karaj, Iran. P.O.Box: 3149779453, Tel: +98 2632563318 , Fax: +98 26 32563329, e-mail: ffiroozeh@ut.ac.ir ORCID ID of corresponding author: https://orcid. org/0000-0002-4026-4110

ACCEPTED: 11 July 2019

\section{INTRODUCTION}

any families of bacteria, unicellular eukaryotes and other organisms live in the human body [1]. Several bacteria such as Bacteroidetes, Firmicutes, and Actinobacteria affect the humans' gut [2]. The term "microbiota," "microflora," or "normal flora" is applied to designate the vast num- ber of microbes that coexist with the host [3]. It is estimated that human microbiota contain 1014 bacterial cells, 10 times more than the number of human cells present in our body [4]. Almost every surface of the human body, from the skin surface to the genitourinary tract, oral cavity, respiratory tract, ear, and the gastrointestinal tract is colonized strongly by different bacteria species $[3,5,6]$. The most heavily colonized 
organ is the gastrointestinal tract (GIT) which hosts a wide microbial ecosystem; the colon alone contains over $70 \%$ of all the microbes in the human body [4]. Intestinal microbial flora play a key role in human health and very important functions are attributed to them including: production of different nutrition for their host, preventing the placement of pathogenic bacteria in intestinal mucosa, protection of epithelial cells barriers, precise maturity of the immune system, the effects on metabolism of the body, and many other important actions [7]. GIT consists of the entire digestive system from the stomach to the anus. The colon is the preferred place for bacteria colonization. GIT is also rich in many molecules that can be used as nutrients by microbes. Therefore, different harmful and beneficial bacteria can heavily colonize the GIT [8]. The gastrointestinal tract mucosa is constantly exposed to a medium that is full of external materials; consists of food particles and microbial antigens [9]. Specific variations in the intestinal ecosystem might relate to the advancement of a specific disease. The imbalanced gut bacteria have been investigated in illnesses including inflammatory bowel disease, antibiotic-associated diarrhea, colon cancer, hypercholesterolemia, and other intestinal and extra-intestinal disorders and diseases [10]. The studies have shown that modification of the gut microbiome in order to achieve a balance in the normal flora of the intestine could be very beneficial in maintaining the health of the human [11]. Lactic acid bacteria, belonging to the genus Lactobacillus and Bifidobacterium, had a positive influence on health. Hence, the study of the use of these bacteria (so-called "probiotics") for the treatment and prevention of diseases should be beneficial. Probiotics were studied in different regions for the treatment of various diseases [5]. Researches showed that probiotics were used to cure microecological problems of acute and chronic diseases such as gastrointestinal, gynaecological cardiovascular and metabolic disorders [12]. In addition, most studies have shown the effects of probiotics on the treatment of digestive diseases like acute diarrhea in newborns and adults [13]. Recent evidence suggest that consumption of probiotics, prebiotics and proper diet has a significant effect on the digestive system; moreover, they reduce and improve symptoms of disorders such as depression, and stress [14]. Probiotics also play an important role in the prevention and treatment of cancers and liver diseases and growth of tumoral cells derived from carcinogens [15]. Further research is needed to better understand the underlying mechanisms and confirm the role of probiotics in preventing and treating various types of diseases. The purpose of this study is to review previous stud- ies on the effects of probiotics on human health and various diseases.

\section{PROBIOTICS}

The most common probiotic microorganisms are divided into three groups of bacteria, fungi and yeasts, some of these microorganisms are selective strains of Lactobacillus and Bifidobacterium bacteria, although strains of Enterococcus, and Escherichia coli are also used for this purpose [5, 16] (Table 1). Among the yeasts, Saccharomyces cerevisiae, and Saccharomyces boulardi can be mentioned [5]. Most of the bacteria of Lactobacillus and Bifidobacterium were recognized as safe [17]. Although lactic acid producing bacteria, except for Streptococcus and Enterococcus, are rarely harmful for humans and animals and their use has long been proven to produce food products without causing adverse effects [5]. Most probiotics are common parts of the human intestinal tract and are consumed in large quantities in functional foods. For example, the population of Bifidobacterium in the human gut can be 1011 cells $/ g$ of intestinal contents [6].

\section{PREBIOTICS}

Prebiotics are certain nutrients that are not easily digested or are less digested in the gastrointestinal tract but selectively stimulate the growth and activity of probiotics and beneficial bacteria in the gut [17]. Low-digestible carbohydrates (LDCs) are an example of known prebiotics, leading to the growth of bifidobacteria and some gram-positive bacteria in the small intestine [12]. Lactolose, galacto-oligosaccharides, fructooligosaccharide, inulin and their hydrolyzed metabolites, maltooligosaccharides are among the prebiotics that are commonly used in human nutrition [12]. The final products of carbohydrate metabolism are short-chain fatty acids (such as acetate, butyrate and propionate) that are used as host energy sources consumed by host organisms. Typically, oligosaccharides, inulin, and oligo-fructans are found in chicory, onion, garlic, asparagus, turnips, leeks, bananas and tomatoes [12]. These prebiotics are produced and marketed in three ways by extraction of plant material, microbial or enzymatic synthesis and enzymatic hydrolysis of polysaccharides in industrial quantities [18].

\section{SYNBIOTICS}

Gibson introduced the synbiotic as the combination of probiotics and prebiotics [19]. Synbiotics influ- 
ence the host health by promoting the survival of live microbial dietary supplements in the intestine [20]. Synbiotics were developed to overcome the probiotic survival problems. It seems that the use of synbiotics increases the survival of probiotic bacteria during passage through the intestine [21]. Different factors such as $\mathrm{pH}, \mathrm{H}_{2} \mathrm{O}_{2}$, organic acids, oxygen and moisture stress, affect the viability of probiotics [22]. The probiotic strains which are usually used in the formulation of synbiotics consist of Lacbobacilli, Bifidobacteria spp, S. boulardii, B. coagulans, and the most prebiotics are comprised of oligosaccharides like fructooligosaccharide (FOS), galactooligosaccharide (GOS), xyloseoligosaccharide (XOS), inulin, and natural prebiotics such as chicory roots [23]. The health advantages of synbiotics utilization by humans are: II) enhanced rates of lactobacilli and bifidobacteria II) balanced gut microbiota, III) ameliorate of liver function in patients with liver disorders, IV) enhancement of immunomodulating ability, VI) decreased frequency of nosocomial infections in hospitalized patients, etc [24].

\section{IMPORTANCE OF PROBIOTICS ON HUMAN HEALTH}

Research has shown that probiotics are used to cure micro-ecological problems of acute and chronic diseases such as gastrointestinal, gynaecological, cardiovascular and metabolic disorders [12]. Probiotic bacteria, by preserving the intestinal microflora and controlling pathogenic microorganisms, reduce the risk of foodborne diseases. Probiotics affect innate, humoral and cellular immune systems as well. Studies have shown that probiotics, by increasing the concentration of globulins, the number and activity of neutrophils and also, by reducing gut microflora such as coliforms, strengthen the immune system and prevent the development of various metabolic and infectious diseases [25].

\section{THE EFFECT OF PROBIOTICS ON THE TREATMENT OF DIGESTIVE DISEASES}

Most studies have shown the effects of probiotics on the treatment of digestive diseases like acute diarrhea in newborns. Allen et al. (2004) reviewed 23 articles on the treatment of infectious diarrhea with probiotics and concluded that probiotics are an adjunctive treatment for infectious diarrhea in children and adults and can be used in conjunction with oral rehydration [13]. Correa et al. (2005) in a clinical trial showed that application of Bifidobacterium lactis and Streptococcus thermophilus is effective in preventing of antibiotic-associated diarrhea in neonates [26]. Saccharomyces bullaria is also used as an effective medication in the treatment of antibiotic-induced diarrhea in some countries [27]. In a study, Guerra et al. (2011) showed that using probiotic yoghurt (containing bifidobacterium longum) improved defecation frequency and abdominal pain in children with functional constipation compared to the control yoghurt [28]. A large number of controlled randomized trials and meta-analyses were conducted on the efficacy of probiotics in preventing antibiotic-associated diarrhea (ADD) and Clostridium difficile-associated diarrhea (CDAD) in children and adults [29-31]. Overall 23 randomized and controlled trials in 4213 patients including adults and children have been performed. The analysis showed that the probiotics can reduce the risk of developing CDAD by $64 \%$, and Saccharomyces bullaria or Lactobacillus grammonosus GG are effective in preventing CDAD following antibiotic therapy [29-31].

\section{THE EFFECT OF PROBIOTICS ON DEVELOPMENT OF CANCERS}

Since uncontrolled cell proliferation and its resistance to programmed death are a major characteristic of cancer cells, the factors that cause apoptosis can be recognized as an anticancer agent. However, resistance to chemotherapy has been a main problem in recent decades. For at least half of the patients with cancer, a certain diet is recommended. In this regard, many of the nutritional compounds and natural products and their relationship with human health attracted the attention of many scientists. The anti-cancer effects of certain probiotics have been documented [15]. In many cases, cancer is created by activating abnormal genes that encode proteins that control cell growth and division. Recent evidence suggests that probiotics can be used to prevent the growth of tumoral cells derived from carcinogens [32]. Kim et al. (2003) investigated the effects of the cell components of ten different probiotic species on eleven types of cancer. The results of these studies indicated that probiotics were effective in inhibiting cancer cells and this effect is attributed to their peptidoglycans [33]. Other studies suggest that induction of apoptosis in human breast cancer cells is due to their in vitro phagocytosis by the yeast [34]. In order to investigate the effect of probiotics on mutagenicity, in a study, Mobarez et al. (2007) observed that living cells of Lactobacillus acidophilus and Lactobacillus bulgaricus, isolated from yoghurt, have a strong antimutagenic activity (64-98\%) against 2-Nitrofluorene mutagen [35]. However, the anti-mutagenic activity was reduced about $47-36 \%$ by heat $\left(100^{\circ} \mathrm{C}\right.$ for 15 minutes) in these cells which indicates that antimutagenic activity largely depends on the survival 
of probiotics [35]. The formation of reactive oxygen species (ROSs) is mainly involved in development of cancers such as colon cancer by creating damage to the DNA. In a laboratory study, Koller et al. (2008) evaluated the detoxification of ROSs using several probiotic species derived from gastrointestinal microbial flora and commercial probiotic products [36]. The researchers multiplied the colon cells extracted from humans and eventually exposed them to 5-Hydroxy2-methyl-1, 4-naphthoquinone and hydrogen peroxide. The results showed that L.acidophilus VM33, Lactobacillus plantarum VM40, Lactococcus lactis VM25, Lactobacillus casei VM33 and Streptococcus thermophilus VM44 reduced the DNA damage caused by 5 -Hydroxy-2-methyl-1, 4-naphthoquinone and hydrogen peroxide [36]. Boniadi and colleagues showed that Saccharomyces cerevisiae and Saccharomyces boulardii inhibit the invitro growth of myeloid cancer cells [37]. An \& $\mathrm{Ha} \mathrm{(2016)}$ in a laboratory study showed that if FU-5 resistant colorectal cancer cells treated with Lactobacillus plantarum, some features of these cells, including the expression of specific cancer markers (CD44, 133, 166, and ALDH1), were inhibited [38]. Further research is needed to better understand the underlying mechanisms and confirm the role of probiotics in preventing and treating various types of cancers.

\section{THE EFFECT OF PROBIOTICS ON DEPRESSION}

Nutrition psychiatry is a branch of psychiatry that examines the relationship between dietary patterns and psychiatric disorders and probiotics have been proposed as the new therapeutic strategy for modulating the nervous system in the face of stress and depression [14]. Consumption of probiotics, prebiotics and proper diet have the significant effects on the health of the digestive system and has reduced and improved symptoms of depression, stress and many digestive diseases [39]. The daily consumption of probiotics in stressed rats causes a significant decrease in the serum adrenocorticotropic hormone (ACTH) levels, which means reducing stress and subsequently reducing depression [40]. In addition, using probiotics prevents severe weight loss in rats exposed to severe stress, which emphasizes the effects of probiotics on stress [40]. Probiotics have the significant effects on the improvement of depressive symptoms through increasing the serotonin levels and reducing inflammation [41]. Research has shown that probiotics can affect the cortisol and serotonin levels in the gastrointestinal tract through the microbiota-gut-brain axis and improve the several parameters such as mood and insomnia in patients with depression [41-43]. Studies by Wallace and col- leagues on depression in humans have shown that depression increases the levels of pro-inflammatory cytokines, such as interleukin 6 (IL-6), Interleukin 1 beta (IL-1 $\beta$ ), Interferon gamma (IFNY) and C-reactive protein (CRP), which leads to an increase in the symptoms of the disease [44]. The increased levels of inflammatory cytokines reduce the level of neurotransmitter precursors and alter their metabolism. This inflammation also increases the permeability of the intestine, and allows the entry of toxic substances and other waste materials into the bloodstream. These gut-derived endotoxins contain the glycoprotein molecules of the outer membrane of gram-negative bacteria that activate the immune system via Toll-like receptor 4 (TLR4) [44].

In another study, Kawai et al. showed that probiotics have therapeutic effects on the nervous system and may reduce the inflammation by creating integrity in the gastrointestinal membrane and reducing the permeability of leaked endotoxins into the bloodstream [45]. Finally, this reducing inflammation regulates the gut-brain axis and neurotransmitters activity [45]. An altered profile of gut microbiota in irritable bowel syndrome (IBS) which is a stress-related disorder of microbiota-gut-brain axis has been proven [46]. A significant number of patients with IBS are also suffering from depression. In these patients an altered Firmicutes: Bacteroidetes ratio has been illustrated [46]. It has been shown that in mice treated with prebiotics such as alacto-oligosaccharides (GOS) the increased intestinal permeability what is happening in IBS, has improved [46].

\section{THE EFFECTS OF PROBIOTICS ON LIVER DISEASES}

Probiotics also play an important role in the prevention and treatment of liver disease. In a study in rats, Savcheniuk et al. (2014) showed that administration of various probiotics during the life of newborns could significantly reduce the risk of fatty liver and obesity [47]. In a survey, on rats with fatty liver, Ritz et al. (2014) assessed the effect of probiotic (Lactobacillus rhamnosus) administration for 8 weeks and stated that this treatment reduced inflammation and liver enzymes [48]. In an experimental study by $\mathrm{Ma}$ et al. (2008) with mouse model, the beneficial effects of the probiotic mix (VSL \# 3) on fat profile, insulin resistance, weight gain and hepatic steatosis have been documented [49]. In another study by Seo et al. (2013), a significant decrease in the lipid accumulation in the liver of rats was achieved following administration of probiotics for 12 weeks [50]. In addition, significant results have been reported on the improvement of lipid metabolism in this group of probiotic-treated rats at the molecular level [50]. In a study 
conducted by Endo et al. (2013) in rats, administration of probiotics (butyrate-producing microorganisms, Clostridium butyricum MIYAIRI 588) significantly reduced or stopped the development of fatty liver [51]. Nonalcoholic fatty liver disease (NAFLD) is the situation that known through excessive triglycerides accumulation in the liver [52]. The disease may lead to fibrosis, cirrhosis, and hepatocellular carcinoma. In a study, Sepideh et al. (2015) evaluated the influences of probiotic (Multi-strain) treatment on glycemic and inflammatory indexes in 42 patients with nonalcoholic fatty liver disease (NAFLD) for 8 weeks. They stated that consumption of probiotic significantly reduced tumor necrosis factor alpha (TNF- $\alpha$ ), IL-6, fasting blood sugar (FBS), insulin and insulin resistance. In their study, fat profile was not investigated [52]. Zhang et al. (2015) declared that probiotics could inhibit steatosis and liver damage in the NAFLD [53]. Liang et al. (2014) also found that the pathogenesis of NAFLD is due to hepatic natural killer T (NKT) cells and glycolipids of probiotics may alter the function of hepatic NKT cells which led to reduction of steatosis [54].

\section{CONCLUSION}

Eventually, probiotics are living microbial supplements that are added to the diet and have beneficial effects on the host by improving the balance of the intestinal microflora. Researches showed that probiotics used to cure micro-ecological problems due to acute and chronic diseases and metabolic disorders. Recent evidences suggest that consumption of probiotics, prebiotics and proper diet have the significant effects on the health of the digestive system and has reduced and improved symptoms of depression, stress and many other diseases like Alzheimer's, heart and diabetes diseases. Probiotics also play an important role in the prevention and treatment of cancers, liver diseases and growth of tumoral cells. Further research is needed to better understand the underlying mechanisms and confirm the role of probiotics in preventing and treating various types of cancers and other diseases.

Disclosure summary: The authors have nothing to disclose.

\section{REFERENCES}

1. Kunz C, Kuntz S, Rudloff S. Intestinal flora. Adv Exp Med Biol 2009; 639:67-79.

2. Morelli L. Postnatal development of intestinal microflora as influenced by infant nutrition. J Nutr 2008; 138(9):1791S-5S.

3. Neish AS. Microbes in gastrointestinal health and disease. Gastroenterology 2009; 136(1):65-80.
4. Ley RE, Peterson DA, Gordon JI. Ecological and evolutionary forces shaping microbial diversity in the human intestine. Cell 2006; 124(4):837-48.

5. Vyas U, Ranganathan N. Probiotics, prebiotics, and synbiotics: gut and beyond. Gastroenterol Res Pract 2012; 2012:872716.

6. Quigley EM. Gut bacteria in health and disease. Gastroenterol Hepatol 2013; 9(9):560-9.

7. Firoozeh F. Gut microbiome and human health. Int J Enteric Pathog 2019; 7(2):30.

8. Canny GO, McCormick BA. Bacteria in the intestine, helpful residents or enemies from within? Infect Immun 2008; 76(8):3360-73.

9. Di Mauro A, Neu J, Riezzo G et al. Gastrointestinal function development and microbiota. Ital J Pediatr 2013;39:15.

10. Dudek-Wicher RK, Junka A, Bartoszewicz M. The influence of antibiotics and dietary components on gut microbiota. Prz Gastroenterol 2018; 13(2):85-92.

11. Sánchez B, Delgado S, Blanco-Míguez A et al. Probiotics, gut microbiota, and their influence on host health and disease. Mol Nutr \& Food Res 2017; 61(1):1-15.

12. Markowiak $P$, Śliżewska K. Effects of probiotics, prebiotics, and synbiotics on human health. Nutrients 2017;9(9):1-30.

13. Allen SJ, Okoko B, Martinez E et al. Probiotics for treating infectious diarrhoea. Cochrane Database of Syst Rev 2004; 2: $\mathrm{CD} 003048$

14. Logan AC, Jacka FN. Nutritional psychiatry research: an emerging discipline and its intersection with global urbanization, environmental challenges and the evolutionary mismatch. J Physiol Anthropol 2014; 33:22.

15. Daniluk U. Probiotics, the new approach for cancer prevention and/or potentialization of anti-cancer treatment? J Clin Exp Oncol 2012; 1:2.

16. Fijan S. Microorganisms with claimed probiotic properties: an overview of recent literature. Int J Environ Res Public Health 2014; 11(5):4745-67.

17. George Kerry R, Patra JK, Gouda S et al. Benefaction of probiotics for human health: A review. J Food Drug Anal 2018;26(3):927-39.

18. Figueroa-González I, Quijano G, Ramírez G et al. Probiotics and prebiotics--perspectives and challenges. J Sci Food Agric 2011;91(8):1341-8.

19. de Vrese M, Schrezenmeir J. Probiotics, prebiotics, and synbiotics. Adv Biochem Eng Biotechnol 2008; 111:1-66.

20. Cencic A, Chingwaru W. The role of functional foods, nutraceuticals, and food supplements in intestinal health. Nutrients 2010; 2(6):611-25.

21. Peña AS. [Intestinal flora, probiotics, prebiotics, symbiotics and novel foods]. Rev Esp Enferm Dig 2007; 99(11):653-8.

22. Romeo J, Nova E, Wärnberg $\mathrm{J}$ et al. Immunomodulatory effect of fibres, probiotics and synbiotics in different life-stages. Nutr Hosp 2010; 25(3):341-9.

23. Pandey KR, Naik SR, Vakil BV. Probiotics, prebiotics and synbiotics- a review. J Food Sci Technol 2015; 52(12):7577-87.

24. Zhang MM, Cheng JQ, Lu YR et al. Use of pre-, pro-and synbiotics in patients with acute pancreatitis: a meta-analysis. World J Gastroenterol 2010; 16(31):3970-8.

25. Saad N, Delattre C, Urdaci M et al. An overview of the last advances in probiotic and prebiotic field. Lebensm Wiss Techno 2013; 50(1), 1-16.

26. Corrêa NB, Péret Filho LA, Penna FJ et al. A randomized formula controlled trial of Bifidobacterium lactis and Streptococcus thermophilus for prevention of antibiotic-associated diarrhea in infants. J Clin Gastroenterol 2005; 39(5):385-9. 
27. Mahmoudi R, Fakhri O, Farhoodi A et al. A Review on probiotic dairy products as functional foods reported from Iran. Int J Food Nutr Saf 2015; 6(1): 1-12.

28. Guerra PV, Lima LN, Souza TC et al. Pediatric functional constipation treatment with Bifidobacterium-containing yogurt: a crossover, double-blind, controlled trial. World J Gastroenterol 2011; 17(34):3916-21.

29. Goldenberg JZ, Ma SS, Saxton JD et al. Probiotics for the prevention of Clostridium difficile-associated diarrhea in adults and children. Cochrane Database Syst Rev 2013;(5):CD006095.

30. Szajewska H, Canani RB, Guarino A et al. Probiotics for the prevention of antibiotic-associated diarrhea in children. $\mathrm{J} \mathrm{Pe}$ diatr Gastroenterol Nutr 2016;62(3):495-506.

31. Pattani R, Palda VA, Hwang SW et al. Probiotics for the prevention of antibiotic-associated diarrhea and Clostridium difficile infection among hospitalized patients: systematic review and meta-analysis. Open Med 2013;7(2):e56-67.

32. Parvez S, Malik KA, Ah Kang $S$ et al. Probiotics and their fermented food products are beneficial for health. J Appl Microbiol 2006; 100(6), 1171-85.

33. Kim JY, Woo HJ, Kim YS et al. Cell cycle dysregulation induced by cytoplasm of Lactococcus lactis ssp lactis in SNUC2A, a colon cancer cell line. Nutr Cancer 2003; 46(2): 197-201.

34. Ghoneum M, Wang L, Agrawal S et al. Yeast therapy for the treatment of breast cancer: anude mice model study. In Vivo 2007; 21(2):251-8.

35. Mobarez AM., Doust RH, Hassan ZM et al. Antimutagenic effect of Lactobacillus acidophilus and Lactobacillus bulgaricus isolated from Iranian yoghurt on 2-nitrofluorene. Res J Microbiol 2007;2(6):524-9

36. Koller VJ, Marian B, Stidl R et al. Impact of lactic acid bacteria on oxidative DNA damage in human derived colon cells. Food Chem Toxicol 2008; 46(4): 1221-9.

37. Boniadi F, Tukmechi A, Nejati V. Comparative study on the effect of obtained cell wall and cytoplasmic fraction from Saccharomyces cerevisiae and Saccharomyces boulardii on K562cell line. Pharm Sci 2012; 18(1):69-78.

38. An J, Ha EM. Combination therapy of Lactobacillus plantarum supernatant and 5 -fluouracil increases chemosensitivity in colorectal cancer cells. J Microbiol Biotechnol 2016; 26(8):1490-503.

39. Dinan TG, Stanton C, Cryan JF. Psychobiotics: a novel class of psychotropic. Biol Psychiatry 2013;74(10):720-6.

40. Soltani Z, Jafari P, Azadegan Ghomi HR. The effect of lactobacillus casei TD2, native Iranian probiotic, on corticosterone hormone secretion in male Wistar rats under chronic restraint stress condition. J Biol Sci 2014;7(1):65-72.

41. McEwen BS. Physiology and neurobiology of stress and adaptation: central role of the brain. Physiol Rev 2007; 87(3):873-904.

42. Paterson LM, Nutt DJ, Wilson SJ. NAPSAQ-1: national patient sleep assessment questionnaire in depression. Int $\mathrm{J}$ Psychiatry Clin Pract 2009; 13(1):48-58.

43. Jackson ML, Butt $H$, Ball $M$ et al. Sleep quality and the treatment of intestinal microbiota imbalance in chronic fatigue syndrome: a pilot study. Sleep Sci 2015; 8(3):124-33.
44. Wallace CJK, Milev R. The effects of probiotics on depressive symptoms in humans: a systematic review. Ann Gen Psychiatry 2017; 16:14.

45. Kawai T, Takeuchi O, Fujita T et al. Lipopolysaccharide stimulates the MyD88-independent pathway and results in activation of IFN-regulatory factor 3 and the expression of a subset of lipopolysaccharide-inducible genes. The J Immunol 2001; 167(10):5887-94.

46. Carroll IM, Ringel-Kulka T, Keku TO et al. Molecular analysis of the luminal- and mucosal-associated intestinal microbiota in diarrhea-predominant irritable bowel syndrome. Am J Physiol Gastrointest Liver Physiol 2011; 301(5):G799-807.

47. Savcheniuk O, Kobyliak N, Kondro M et al. Short-term periodic consumption of multiprobiotic from childhood improves insulin sensitivity, prevents development of non-alcoholic fatty liver disease and adiposity in adult rats with glutamate-induced obesity. BMC Complement Altern Med 2014; 14:247.

48. Ritze $Y$, Bárdos G, Claus A et al. Lactobacillus rhamnosus GG protects against non-alcoholic fatty liver disease in mice. PLoS One 2014; 9(1):e80169.

49. Ma X, Hua J, Li Z. Probiotics improve high fat diet-induced hepatic steatosis and insulin resistance by increasing hepatic NKT cells. J Hepatol 2008; 49(5):821-30.

50. Seo M, Inoue I, Tanaka M et al. Clostridium butyricum MIYAIRI 588 improves high-fat diet induced non-alcoholic fatty liver disease in rats. Dig Dis Sci 2013; 58(12):3534-44.

51. Endo H, Niioka M, Kobayashi $\mathrm{N}$ et al. Butyrate-producing probiotics reduce nonalcoholic fatty liver disease progression in rats: new insight into the probiotics for the gut-liver axis. PLoS One 2013; 8(5):e63388.

52. Sepideh A, Karim P, Hossein A et al. Effects of multistrain probiotic supplementation on glycemic and inflammatory indices in patients with nonalcoholic fatty liver disease: a double- blind randomized clinical trial. J Am Coll Nutr 2016;35(6):500-5.

53. Zhang M, Wang C, Wang C et al. Enhanced AMPK phosphorylation contributes to the beneficial effects of Lactobacillus rhamnosus GG supernatant on chronic-alcohol-induced fatty liver disease. J Nutr Biochem 2015; 26: 337-44.

54. Liang S, Webb T, Li Z. Probiotic antigens stimulate hepatic natural killer T cells. Immunology 2014; 141(2): 203-10.

55. Villena J, Kitazawa H. Probiotic microorganisms: a closer look. Microorganisms 2017; 5(2). pii: E17.

56. Selle K, Klaenhammer TR. Genomic and phenotypic evidence for probiotic influences of Lactobacillus gasseri on human health. FEMS Microbiol Rev 2013; 37(6):915-35.

57. Elshaghabee FMF, Rokana N, Gulhane RD et al. Bacillus as potential probiotics: status, concerns, and future perspectives. Front Microbiol 2017; 8:1490.

58. Ianiro G, Rizzatti G, Plomer M et al. Bacillus clausii for the treatment of acute diarrhea in children: a systematic review and meta-analysis of randomized controlled trials. Nutrients 2018; 10(8). pii: E1074.

59. Salas-Jara MJ, Ilabaca A, Vega M et al. Biofilm forming Lactobacillus: new challenges for the development of probiotics. Microorganisms 2016; 4(3). pii: E35. 